\title{
A zero-dimensional topologically nontrivial state in a superconducting quantum dot
}

\author{
Pasquale Marra ${ }^{* 1}$, Alessandro Braggio ${ }^{2}$ and Roberta Citro ${ }^{3}$
}

\section{Full Research Paper}

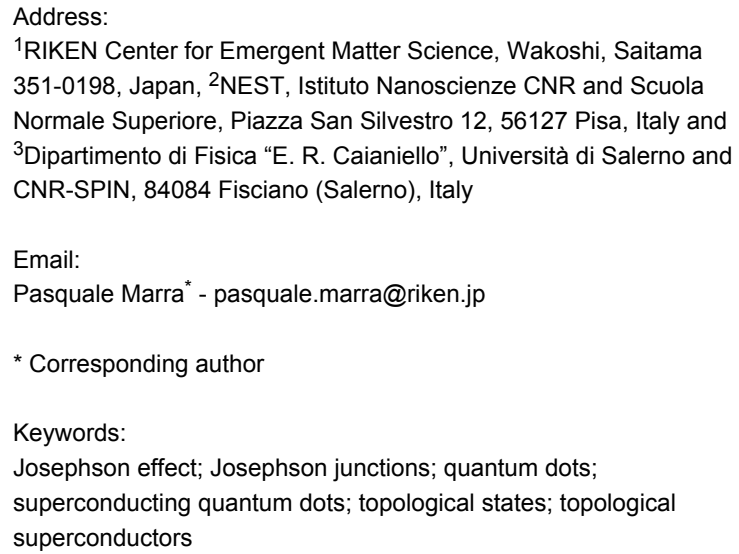

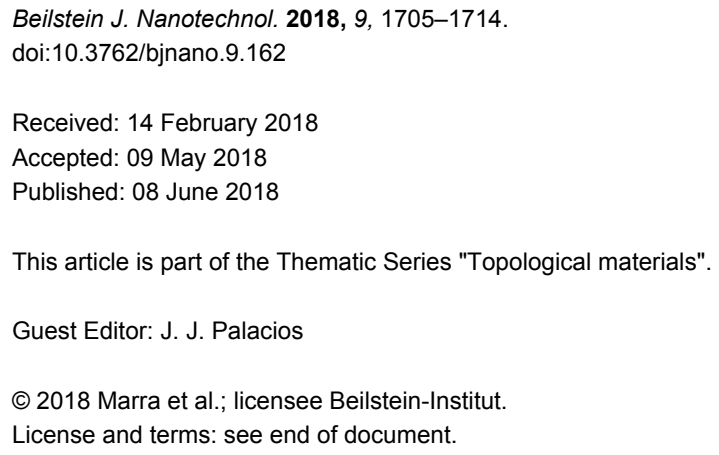

\begin{abstract}
The classification of topological states of matter in terms of unitary symmetries and dimensionality predicts the existence of nontrivial topological states even in zero-dimensional systems, i.e., systems with a discrete energy spectrum. Here, we show that a quantum dot coupled with two superconducting leads can realize a nontrivial zero-dimensional topological superconductor with broken time-reversal symmetry, which corresponds to the finite size limit of the one-dimensional topological superconductor. Topological phase transitions corresponds to a change of the fermion parity, and to the presence of zero-energy modes and discontinuities in the current-phase relation at zero temperature. These fermion parity transitions therefore can be revealed by the current discontinuities or by a measure of the critical current at low temperatures.
\end{abstract}

\section{Introduction}

Since the discovery of the quantum Hall effect $[1,2]$ and the theoretical prediction of Majorana bound states in triplet superconductors [3], a whole new class of novel electronic phases has been theoretically described and experimentally realized, namely, the class of topologically nontrivial states of matter [4-7]. Topological states of matter can be classified in terms of the antiunitary symmetries and dimensionality of the Hamiltonian [7-10]. Analogously to the periodic table of chemical ele- ments in chemistry, this classification has been a general guide to the discovery of novel topological phases in solid-state physics. Moreover, it predicts the existence of nontrivial topological states even in zero dimensions, i.e., in a system with discrete energy spectrum.

A very important class of topological states of matter are topological superconductors: These materials support Majorana 
zero-energy modes at the edges of the system [11-13], which have been proposed as the building block of topological quantum devices [14-20]. The simplest realization of a topological superconductor is the well-known Kitaev chain [3], which can be implemented in a one-dimensional system proximized by a conventional superconductor in the presence of a magnetic field and spin-orbit coupling [21-25]. Moreover, topological superconductors exhibit very distinct features in their transport properties and in particular in their Josephson current [26-49].

In a recent work [50], we have studied the short-size limit of a one-dimensional (1D) topological superconductor with broken time-reversal and chiral symmetries. In this limit, the system turns zero-dimensional (0D), i.e., its energy spectrum is a finite set of discrete energy levels. This $0 \mathrm{D}$ superconductor exhibits topological phase transitions that correspond to variations of the fermion parity and to the occurrence of zero-energy modes that are a linear combination of particle and hole states [50]. These fermion parity transitions can be revealed by discontinuities in the Josephson current-phase relation (CPR) in the zero-temperature limit.

Here we describe the simplest realization of such a 0D topological superconductor, i.e., a quantum dot [51-54] coupled with two superconducting leads in a magnetic Zeeman field, forming a superconductor-quantum dot-superconductor (SC-QD-SC) Josephson junction. Zero-energy modes and the corresponding CPR discontinuities and ground-state parity crossings [55-61] have been recognized as precursors of Majorana modes in the long-wire limit [27,50], and of Floquet-Majorana modes realized in driven quantum dots $[62,63]$. We will analytically derive and discuss the spectrum and the Josephson current of the dot, which agrees with the universal prediction for zero-dimensional systems described in our previous work [50]. This allows us to reinterpret in terms of topological states the different regimes of the dot, which are already discussed in the literature [34,64-68]. We will analyze in detail the relation between the topological properties of the groundstate, the zero-energy modes, and the corresponding CPR discontinuities. We will show that, in this system, a topologically nontrivial state can be induced by a finite Zeeman field that breaks the time-reversal symmetry, even without a finite spin-orbit coupling. The resulting topological transitions coincide with a change of the fermion parity (topological invariant) and can be identified by discontinuities in the CPR and by a measure of the critical current at low temperatures.

\section{Results and Discussion Effective model}

We consider a semiconducting quantum dot in a magnetic field $B$ and coupled with two superconducting leads, as shown in
Figure 1. We assume that the only effect of the magnetic field is the lifting of the spin degeneracy via the Zeeman effect, and we neglect orbital effects of the field. Moreover, we assume that the level spacing of the dot is larger than the Zeeman energy $B$ and larger than the Coulomb interaction $U$ within the dot. Therefore we neglect the contribution of higher energy levels and take into account only the levels $\varepsilon \pm B$ of the Kramers doublet closest to the Fermi energy. Here, $\varepsilon$ is the energy level of the dot in absence of Zeeman field, which can be modified by controlling the gate voltage. This system can be described by a superconducting Anderson impurity model

$$
H=H_{\mathrm{QD}}+\sum_{i=L, R} H_{i}+H_{t_{i}},
$$

where the dot Hamiltonian is given by

$$
\begin{aligned}
H_{\mathrm{QD}} & =\varepsilon\left[\begin{array}{ll}
d_{\uparrow}^{\dagger} & d_{\downarrow}^{\dagger}
\end{array}\right] \cdot\left[\begin{array}{l}
d_{\uparrow} \\
d_{\downarrow}
\end{array}\right]+B\left[\begin{array}{ll}
d_{\uparrow}^{\dagger} & d_{\downarrow}^{\dagger}
\end{array}\right] \cdot \sigma_{z} \cdot\left[\begin{array}{l}
d_{\uparrow} \\
d_{\downarrow}
\end{array}\right] \\
& +U\left(n_{\uparrow}-\frac{1}{2}\right)\left(n_{\downarrow}-\frac{1}{2}\right),
\end{aligned}
$$

where $d_{\uparrow}^{\dagger}, d_{\downarrow}^{\dagger}$ and $d_{\uparrow}, d_{\downarrow}$ are the creation and annihilation operators of the electrons in the dot, $n_{\uparrow}=d_{\uparrow}^{\dagger} d_{\uparrow}$ and $n_{\downarrow}=d_{\downarrow}^{\dagger} d_{\downarrow}$ the number operators, $\varepsilon \pm B$ the two-energy levels of the dot, and $U$ the onsite Coulomb repulsion. We assume hereafter that $e=\hbar=1$.

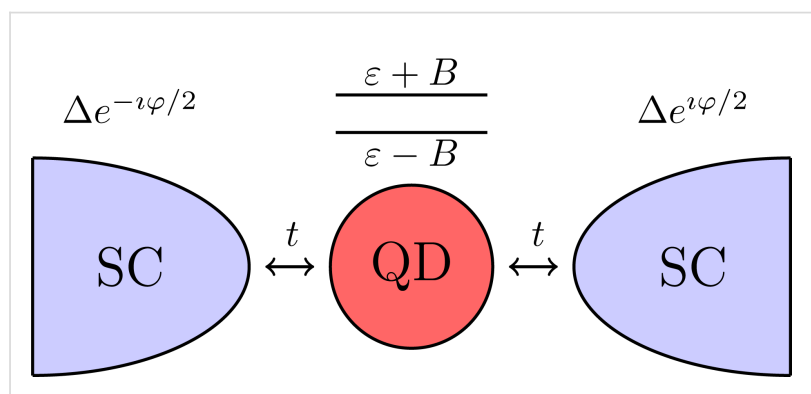

Figure 1: An SC-QD-SC Josephson junction realized by a two-level quantum dot in a magnetic field $B$ and electric gate $\varepsilon$ coupled with two superconducting leads. The two energy levels are respectively $\varepsilon \pm B$. The dot is coupled to the superconducting leads via tunneling junctions with transparency $t$. The Josephson current $I_{\varphi}$ through the dot depends on the gauge-invariant phase difference $\varphi$ between the two superconducting leads.

The Hamiltonians of the two superconducting leads $i=L, R$ are given by 


$$
\begin{aligned}
H_{i} & =\sum_{\mathbf{k}} \varepsilon_{\mathbf{k}}\left[\begin{array}{cc}
c_{\mathbf{k}, i, \uparrow}^{\dagger} & c_{\mathbf{k}, i, \downarrow}^{\dagger}
\end{array}\right] \cdot\left[\begin{array}{c}
c_{\mathbf{k}, i, \uparrow} \\
c_{\mathbf{k}, i, \downarrow}
\end{array}\right] \\
& +\left(\frac{1}{2} \Delta e^{l \varphi_{i}}\left[\begin{array}{cc}
c_{\mathbf{k}, i, \uparrow}^{\dagger} & c_{\mathbf{k}, i, \downarrow}^{\dagger}
\end{array}\right] \cdot\left(l \sigma_{y}\right) \cdot\left[\begin{array}{c}
c_{-\mathbf{k}, i, \uparrow}^{\dagger} \\
c_{-\mathbf{k}, i, \downarrow}^{\dagger}
\end{array}\right]+\text { h.c. }\right),
\end{aligned}
$$

where $c_{\mathbf{k}, i, \uparrow}^{\dagger}, c_{\mathbf{k}, i, \downarrow}^{\dagger}$ and $c_{\mathbf{k}, i, \uparrow}, c_{\mathbf{k}, i, \downarrow}$ are the creation and annihilation operators of electrons in the superconducting lead $i=L, R$ and with momentum $\mathbf{k}, \varepsilon_{\mathbf{k}}$ is the bare electron dispersion with respect to the Fermi level $\varepsilon_{\mathrm{F}}=0, \Delta$ the magnitude of the superconducting gap, and $\varphi_{i}$ the phase of the superconducting gap in the two leads, respectively. Here we assumed a standard BCS $s$-wave pairing and the same bare electron dispersion in the two superconducting leads. In the following we furthermore assume that the bare electron dispersion varies in the interval $[-D, D]$ and that the density of states is $\rho_{0}=1 /(2 D)$ with $2 D$ the total bandwidth.

The tunneling between the dot and the leads is described by the tunnel Hamiltonians, which read

$$
H_{t_{i}}=t \sum_{\mathbf{k}}\left[\begin{array}{ll}
c_{\mathbf{k}, i, \uparrow}^{\dagger} & c_{\mathbf{k}, i, \downarrow}^{\dagger}
\end{array}\right] \cdot\left[\begin{array}{l}
d_{\uparrow} \\
d_{\downarrow}
\end{array}\right]+\text { h.c. }
$$

where $t=t_{L}=t_{R}$ is the transparency of the dot-lead tunneling. We assume that the junction is symmetric and that the tunneling amplitudes do not depend on the electron momenta (wide band limit approximation).

In the limit of a large superconducting gap, i.e., when the gap is larger than the characteristic frequencies of the quantum dot, the degrees of freedom of the leads can be effectively integrated out [34,64-68]. In absence of interactions $(U=0)$ the system can be described by an effective Hamiltonian that reads $[34,64,65,67,68]$

$$
\begin{aligned}
H_{\mathrm{eff}} & =\left[\begin{array}{ll}
d_{\uparrow}^{\dagger} & d_{\downarrow}^{\dagger}
\end{array}\right] \cdot\left(\varepsilon+B \sigma_{z}\right) \cdot\left[\begin{array}{l}
d_{\uparrow} \\
d_{\downarrow}
\end{array}\right] \\
& +\Gamma \cos (\varphi / 2)\left[\begin{array}{ll}
d_{\uparrow}^{\dagger} & d_{\downarrow}^{\dagger}
\end{array}\right] \cdot\left(l \sigma_{y}\right) \cdot\left[\begin{array}{c}
d_{\uparrow}^{\dagger} \\
d_{\downarrow}^{\dagger}
\end{array}\right]+\text { h.c., }
\end{aligned}
$$

where $\varphi=\varphi_{R}-\varphi_{L}$ is the gauge-invariant phase difference between the two leads, and where

$$
\Gamma=4 t^{2} \rho_{0} \arctan \left(\frac{D}{\Delta}\right)
$$

is the effective local superconducting pairing induced by the leads on the dot $[64,65]$. The Hamiltonian (Equation 5) can be written in the Bogoliubov-de Gennes formalism as

$$
H_{\mathrm{eff}}=\Psi^{\dagger} \cdot\left[\begin{array}{cc}
\varepsilon+B \sigma_{z} & \Gamma \cos (\varphi / 2) \imath \sigma_{y} \\
-\Gamma \cos (\varphi / 2) \iota \sigma_{y} & -\varepsilon-B \sigma_{z}
\end{array}\right] \cdot \Psi
$$

where $\Psi^{\dagger}=\left[d_{\uparrow}^{\dagger}, d_{\downarrow}^{\dagger}, d_{\uparrow}, d_{\downarrow}\right]$ and $\Psi=\left[d_{\uparrow}, d_{\downarrow}, d_{\uparrow}^{\dagger}, d_{\downarrow}^{\dagger}\right]^{\top}$ are the Nambu spinors describing the electron-hole pairs in the dot. Notice that our definition of Nambu spinor differs from $[64,65]$, but it will allow us to define the topological invariant using the same formalism used in 1D superconductors.

The spectrum of this effective Hamiltonian is a set of four single-particle states corresponding to two pairs of particle-hole symmetric Andreev levels $\pm E_{\uparrow}$ and $\pm E_{\downarrow}$ with

$$
\begin{aligned}
& E_{\uparrow}=E_{\varphi}+B, \\
& E_{\downarrow}=E_{\varphi}-B,
\end{aligned}
$$

with

$$
E_{\varphi}=\sqrt{\varepsilon^{2}+\Gamma^{2} \cos ^{2}(\varphi / 2)}
$$

which correspond to the eigenstates described by the operators $\bar{d}_{\uparrow \downarrow}^{\dagger}$ defined by the Bogoliubov transformation

$$
\begin{aligned}
& \bar{d}_{\uparrow}^{\dagger}=u d_{\uparrow}^{\dagger}+v d_{\downarrow}, \\
& \bar{d}_{\downarrow}^{\dagger}=u d_{\downarrow}^{\dagger}-v d_{\uparrow},
\end{aligned}
$$

where

$$
\begin{aligned}
& u=\sqrt{\left(1+\varepsilon / E_{\varphi}\right) / 2}, \\
& v=\sqrt{\left(1-\varepsilon / E_{\varphi}\right) / 2},
\end{aligned}
$$

The Bogoliubov factors satisfy the properties $u^{2}+v^{2}=1$, $u^{2}-v^{2}=\varepsilon / E_{\varphi}$, and $u v=\Gamma|\cos (\varphi / 2)| /\left(2 E_{\varphi}\right)$. 
Now we generalize the Hamiltonian (Equation 7) to the case of finite interaction $U>0$. A tedious but elementary calculation gives $\left(n_{\uparrow}-1 / 2\right)\left(n_{\downarrow}-1 / 2\right)=\left(\bar{n}_{\uparrow}-1 / 2\right)\left(\bar{n}_{\downarrow}-1 / 2\right)$ where $\bar{n}_{\uparrow}=\bar{d}_{\uparrow}^{\dagger} \bar{d}_{\uparrow}$ and $\bar{n}_{\downarrow}=\bar{d}_{\downarrow}^{\dagger} \bar{d}_{\downarrow}$ are the number operators corresponding to the eigenstates of the effective Hamiltonian. Therefore the Hamiltonian in the presence of Coulomb interaction $U$ $>0$ can be written in diagonal form as

$$
\bar{H}_{\mathrm{eff}}=\left(E_{\varphi}-\frac{U}{2}\right)\left(\bar{n}_{\uparrow}+\bar{n}_{\downarrow}\right)+B\left(\bar{n}_{\uparrow}-\bar{n}_{\downarrow}\right)+U \bar{n}_{\uparrow} \bar{n}_{\downarrow},
$$

up to a numerical phase-independent constant.

The Hamiltonian eigenstates comprise the vacuum $|00\rangle$, the two single-particle states $|01\rangle$ and $|10\rangle$, and the two-particle state $|11\rangle$ with energies

$$
\begin{gathered}
\bar{E}_{0}=0, \\
\bar{E}_{\downarrow}=E_{\varphi}-U / 2-B, \\
\bar{E}_{\uparrow}=E_{\varphi}-U / 2+B, \\
\bar{E}_{\uparrow \downarrow}=2 E_{\varphi} .
\end{gathered}
$$

Each of these particle states corresponds to a hole state by particle-hole symmetry. The groundstate energy of the superconducting condensate is given by the sum of the single-particle energy levels [69], which yield in this case

$$
E_{\mathrm{GS}}(\varphi)=\left|E_{\varphi}-U / 2-B\right|+\left|E_{\varphi}-U / 2+B\right|
$$

whereas the Josephson current at zero temperature is defined as $I_{\varphi}=-\partial_{\varphi} E_{\mathrm{GS}}(\varphi)$. Notice that for small couplings $U / 2<|\varepsilon|,|\Gamma|$, the only effect of the interaction is to shift the energy of the single-particle levels. For this reason, if the conductance from the dot to the superconductor is relatively large (high dot-lead transparency) and one can consider the effect of interactions as a small perturbation. Therefore, the ground-state properties, such as the topological invariant and the Josephson current at zero temperature, are not affected in the case where $U / 2<\varepsilon$ and $U / 2<\Gamma$, as long as the particle-hole gap remains open and the Andreev levels do not cross.
In absence of interactions $U=0$, the only possible ground states are those with energies

$$
E_{\mathrm{GS}}(\varphi)= \begin{cases}2 E_{\varphi} & \text { for } E_{\uparrow} E_{\downarrow}>0, \\ 2 B & \text { for } E_{\uparrow} E_{\downarrow}<0,\end{cases}
$$

which correspond, respectively, to the cases where the two single-particle levels $E_{\uparrow}$ and $E_{\downarrow}$ have the same sign or opposite sign. We will show that the ground state with energy $2 E_{\varphi}$ is topologically trivial and has a finite Josephson current, whereas the ground state with energy $2 B$ is topologically nontrivial and has a Josephson current that vanishes at zero temperature.

The phase diagram of this system has been already discussed in the literature [34,64-68]. Since we consider here only the weak interacting case, we will not discuss the $0-\pi$ transition driven by the presence of strong interaction. A more thorough discussion of the role of interactions on the 0D topological transition and on the ensuing $\pi$-phase will be addressed in a following research paper. Therefore, we will discuss hereafter only quantum phase transition in the regime of weak interactions in systems which can be described by Equation 7 or Equation 14 for $U=0$. Our findings cannot be applied to $0-\pi$ transitions and to other kinds of quantum phase transitions that may be eventually present in this system, beyond the topological one we discussed.

\section{The particle-hole gap and gapless points}

The particle-hole gap, i.e., the difference between the particle and hole levels closest to the Fermi level, closes if $|B|=E_{\varphi}$. If one defines the two threshold fields $B_{\min }=|\varepsilon|$ and $B_{\max }=\sqrt{\varepsilon^{2}+\Gamma^{2}}$, one can verify that the spectrum is gapped for both small $|B|<B_{\min }$ and large $|B|>B_{\max }$ Zeeman fields. For intermediate fields $B_{\min }<|\mathrm{B}|<B_{\max }$, the energy gap closes at specific values of the gauge-invariant phase $\varphi= \pm \varphi^{*}$ where

$$
\varphi^{*}=\arccos (-\lambda) \quad \text { with } \lambda=1+\frac{2\left(\varepsilon^{2}-B^{2}\right)}{\Gamma^{2}}
$$

where $|\lambda|<1$ if $B_{\min }<|B|<B_{\max }$. We will show that these gapless points define a topological phase transition in the system that corresponds to the appearance of discontinuous drops in the CPR of the junction.

Figure 2 shows the single-particle energy spectrum of the system, i.e., the four particle-hole symmetric Andreev levels $\pm E_{\downarrow}$ and $\pm E_{\uparrow}$, as a function of the gauge-invariant phase differ- 
(a)

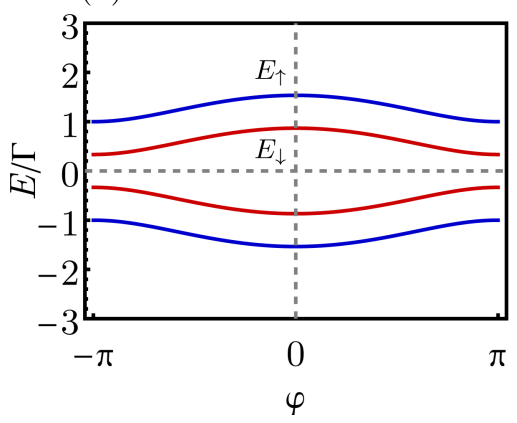

(b)

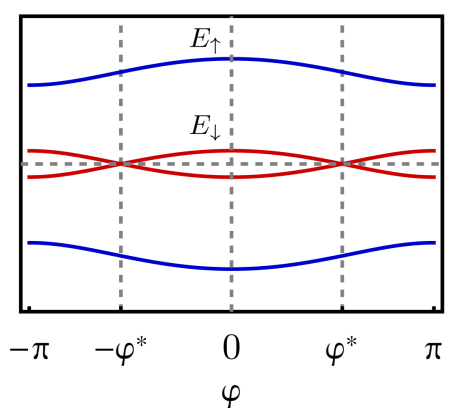

(c)

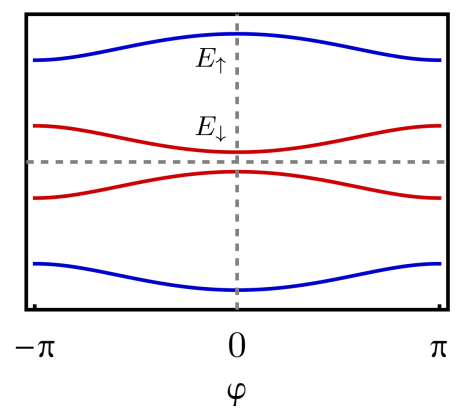

Figure 2: Energy spectrum of a two-level quantum dot coupled with two superconducting leads (SC-QD-SC junction), consisting of a set of four Andreev levels, i.e., two single-particle levels $\pm E_{\uparrow}$ (blue curves) and $\pm E_{\downarrow}$ (red curves), as a function of the gauge-invariant phase difference $\varphi$ between the two superconducting leads. We take $\varepsilon=2 \Gamma / 3$ and $U=0$. The three panels correspond to different values of the Zeeman field: (a) small fields $|B|<B_{\min }$, (b) intermediate fields $B_{\min }<|B|<B_{\max }$, with the particle-hole gap closing at the gapless points $\pm \varphi^{*}$ (see Equation 21), and (c) large fields $|B|>B_{\max }$.

ence $\varphi$. As one can see, the energy spectrum is gapped for small $|B|<B_{\min }$ and large $|B|>B_{\max }$ Zeeman fields, respectively, independently from the phase difference $\varphi$. At intermediate fields $B_{\text {min }}<|B|<B_{\text {max }}$, the particle-hole gap closes at the gapless points $\pm \varphi *$ that satisfy Equation 21. One can verify that the effect of a small Coulomb interaction $U / 2<|\varepsilon|,|\Gamma|$ is a shift of the threshold fields $B_{\min }$ and $B_{\max }$ and of the value of the phases $\pm \varphi *$ where the gap closes.

\section{Topological invariant}

This simple 0D two-level system can realize a topologically nontrivial state that breaks time-reversal symmetry while preserving particle-hole symmetry. This topologically nontrivial state can be seen as the 0D limit of a 1D topological superconductor, and as the minimal model for the system described in [50]. In fact, for finite Zeeman energies $(B \neq 0)$ and superconducting pairing $(\Gamma>0)$, the system is in the Altland-Zirnbauer [7-10] symmetry class D (particle-hole symmetry, broken time-reversal and chiral symmetries). This class is characterized in $0 \mathrm{D}$ by a $\mathbb{Z}_{2}$ topological invariant that is defined in the non-interacting case $U=0$ as the fermion parity of the ground state $[50,70] P=\operatorname{sgn} \operatorname{pf}\left(H_{\text {eff }} l \tau_{x}\right)$, i.e., as the sign of the Pfaffian of the Hamiltonian in Majorana representation ( $\tau_{x}$ is the first Pauli matrix in the particle-hole space). The fermion parity labels the topological inequivalent ground states as a function of the gauge-invariant phase $\varphi$, i.e., the trivial state $P=1$ (even parity) and nontrivial state $P=-1$ (odd parity). The fermion parity of the 0D topological quantum dot described by Hamiltonian (Equation 7) can be evaluated analytically. The square of the Pfaffian of a matrix is equal to the determinant, which is equal to the product of its eigenvalues, and therefore one has $\operatorname{pf}\left(H_{\text {eff }} \iota \tau_{x}\right)^{2}=\operatorname{det}\left(H_{\text {eff }} \iota \tau_{x}\right)=\operatorname{det}\left(H_{\text {eff }}\right)=E_{\uparrow}^{2} E_{\downarrow}^{2}$ due to particle-hole symmetry. A direct calculation of the Pfaffian indeed shows that $\operatorname{pf}\left(H_{\mathrm{eff}} l \tau_{x}\right)=E_{\uparrow} E_{\downarrow}$ and therefore
$P_{\varphi}=\operatorname{sgn}\left(E_{\uparrow} E_{\downarrow}\right)=\operatorname{sgn}\left(E_{\varphi}^{2}-B^{2}\right)=\operatorname{sgn}(\lambda+\cos \varphi)$,

where we used the definition of $\lambda$ given in Equation 21. This equation is a special case of Equation 2 of [50]. Notice that if $B=0$ the time-reversal symmetry is unbroken and the ground state is trivial $P_{\varphi}=\operatorname{sgn}\left(E_{\varphi}^{2}\right)=1$ as expected. As anticipated, the ground state with energy $2 E_{\varphi}$ is topologically trivial, since in this case $E_{\uparrow} E_{\downarrow}>0$, whereas the ground state with energy $2 B$ is topologically nontrivial, since in this case one has $E_{\uparrow} E_{\downarrow}<0$. Therefore, the inversion of the lowest-energy Andreev level corresponds to a topological transition to the nontrivial state. The fermion parity defines the topological phase space of the system, and is completely determined by the gauge-invariant phase $\varphi$ and by the adimensional quantity $\lambda$, as shown in Figure 3. Moreover, since $P=\operatorname{sgn}\left[E_{\uparrow} E_{\downarrow}\right]$, the condition $P_{\varphi} \equiv 0$ corresponds to the gapless points $\varphi= \pm \varphi^{*}$ where zero-energy modes occur (solid line in Figure 3).

At small Zeeman fields $|B|<B_{\min }$ (i.e., $\lambda>1$ ), the system is in the topologically trivial state with even fermion parity $P=1$ for any value of the phase $\varphi$. At large fields $|B|>B_{\max }$ instead (i.e., $\lambda<-1$ ), the system realizes the topologically nontrivial state with odd fermion parity $P=-1$ for any value of the phase $\varphi$. However, for intermediate $B_{\min }<|B|<B_{\max }$ (i.e., $|\lambda|<1$ ) topological transitions occur at the gapless points $\pm \varphi *$ (see Equation 21). In this case the system realizes the trivial or in the nontrivial state (even or odd parity), respectively, for $|\varphi|<\varphi^{*}$ and $|\varphi|>\varphi^{*}$ in the interval $\varphi \in[-\pi, \pi]$, as one can see in Figure 3. The two gapless points $\pm \varphi *$ therefore correspond to a quantum phase transition where the fermion parity of the ground state changes from trivial to nontrivial. Note that for $|B|=B_{\min }$ and for $|B|=B_{\max }$ (i.e., $|\lambda|=1$ ) no topological transition occurs, and the system is, respectively, in the trivial or 


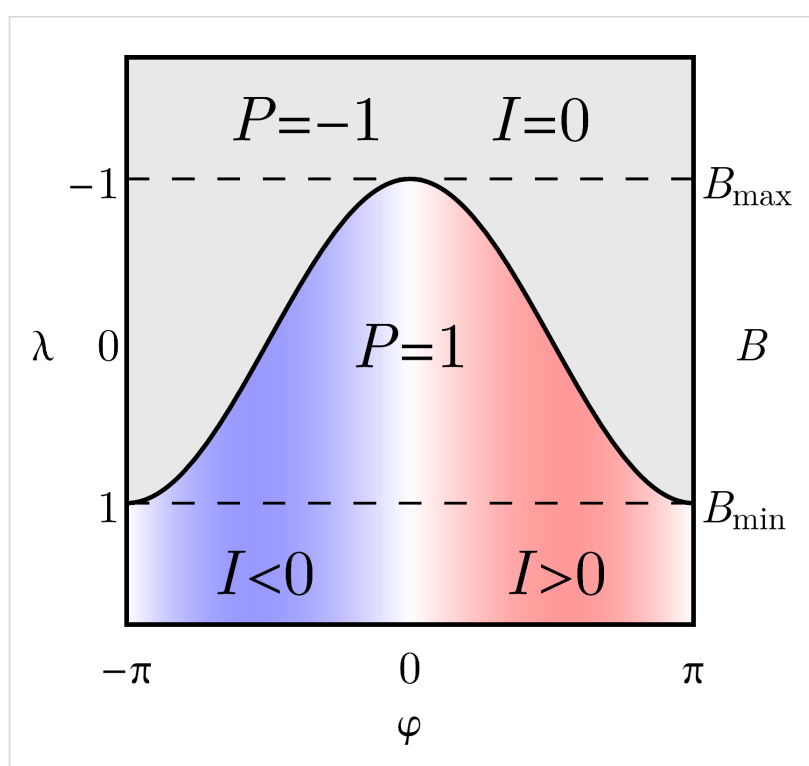

Figure 3: Topological phase space of a OD topological superconductor realized by a quantum dot coupled with two superconducting leads (SC-QD-SC junction). The system realizes, respectively, a trivial state $P=1$ for small Zeeman fields $|B|<B_{\min }$ (i.e., $\lambda>1$ ), and a nontrivial state $P=-1$ for large fields $|B|>B_{\max }$ (i.e., $\lambda<-1$ ). The Josephson current vanishes in the nontrivial state. Topological transitions coincides with the occurrence of zero-energy modes at $\pm \varphi^{*}= \pm \arccos (-\lambda)$ (solid line) for intermediate fields $B_{\min }<|B|<B_{\max }$ (i.e., $|\lambda|<1$ ). In this case the system is in its trivial $P=1$ and nontrivial $P=-1$ state respectively for $|\varphi| \lessgtr \varphi^{*}$ within the interval $\varphi \in[-\pi, \pi]$.

nontrivial gapped state with the exceptions of the single gapless point $\varphi^{*}=\pi$ or $\varphi^{*}=0$, respectively.

The particle-hole gap can also close in absence of a Zeeman field if $\varepsilon=0$. For $B=\varepsilon=0$ (which gives $\lambda=1$ ) the gap closes at $\varphi^{*}=\pi$. In this case the time-reversal symmetry is unbroken, and the system is gapped and topologically trivial for any value of the phase $\varphi \neq \pi$.

The topological phase space derived in the case of a superconducting quantum dot is universal for the class of zero-dimensional superconductors. It coincides in fact with the topological phase space in Figure 2a of [50], where it was derived in the more general case of a zero-dimensional quantum system (short-size regime) with an arbitrary number of energy modes. The topological phases can be defined also in the case of small Coulomb interactions as long as the particle-hole gap remains open. In this case in fact the topological invariant cannot change, since the phase with small interaction $U>0$ can be transformed with the non-interacting phase $U=0$ by a smooth transformation without closing the gap.

It is important to note that in the $0 \mathrm{D}$ case (differently from the 1D case) topological states can be realized without spin-orbit coupling. This is because topological states in the symmetry class D are enforced by the presence of the superconducting coupling (particle-hole symmetry) and the Zeeman field (which breaks the time-reversal symmetry). The gap opening, in this case, is guaranteed in general by the gap induced by finite size effects or eventually by interactions.

\section{Josephson current-phase discontinuities}

In our previous work [50], we have found the general relation between the topological invariant of a 0D topological superconductor and the discontinuities of the Josephson current-phase relation (CPR). The topological phase transition between the trivial ( $P=1$, even fermion parity) and the nontrivial state ( $P=-1$, odd fermion parity) corresponds to the emergence of a discontinuity in the Josephson CPR at zero temperature. In this case, the current is proportional to the phase-derivative of the total energy of the superconducting condensate [69,71], which is given by the sum of the positive energy levels $\left|E_{\uparrow}\right|+\left|E_{\downarrow}\right|$. Hence, the Josephson current is equal to $-2 \partial_{\varphi} E_{\varphi}$ in the trivial groundstate with energy $E_{\mathrm{GS}}(\varphi)=2 E_{\varphi}$, whereas it vanishes in the nontrivial groundstate with energy $E_{\mathrm{GS}}(\varphi)=2 B$ (see Equation 20). The CPR at zero temperature is therefore given by

$$
I_{\varphi}=-\left(1+P_{\varphi}\right) \partial_{\varphi} E_{\varphi}=\left(1+P_{\varphi}\right) \frac{\Gamma^{2} \sin \varphi}{4 E_{\varphi}} .
$$

In the topologically trivial state $(P=1)$ at low fields $|B|<B_{\min }$, the two energy levels $E_{\uparrow}$ and $E_{\downarrow}$ contribute equally to the Josephson current and one has $I_{\varphi}=-2 \partial_{\varphi} E_{\varphi}$. However, when the fermion parity changes, one of the energy level crosses the particle-hole gap, and its contribution to the current changes its sign.

Therefore, in the topologically nontrivial state $(P=-1)$ at high fields $|B|>B_{\max }$ the Josephson current in Equation 23 vanishes since the contributions from the two energy levels $E_{\uparrow}$ and $E_{\downarrow}$ cancel each other. Moreover, as one can see from Equation 23, for intermediate fields $B_{\min }<|B|<B_{\max }$, (i.e., $|\lambda|<1$ ) the CPR exhibits a discontinuity between the trivial state with $I= \pm 2 \Gamma^{2} \sin \varphi^{*} /\left[4 E_{\varphi^{*}}\right]$ to the nontrivial one with $I=0$ at the gapless points $\pm \varphi^{*}$ which is equal to

$$
\Delta I=\frac{\Gamma^{2} \sqrt{1-\lambda^{2}}}{2|B|},
$$

which is a special case of Equation 3 of [50]. The discontinuity is a consequence of the crossing at zero-energy of the lowestenergy level with linear phase dispersion. The discontinuity in Equation 24 can be also calculated directly using Equation 3 of [50], which can be rewritten as 


$$
\Delta I=\left.2 \frac{\left|\partial_{\varphi} \operatorname{pf}\left(\mathcal{H}_{\mathrm{eff}} \iota \tau_{x}\right)\right|}{\sqrt{\left|\operatorname{pdet}\left(\mathcal{H}_{\mathrm{eff}}\right)\right|}}\right|_{\varphi=\varphi^{*}},
$$

where $\operatorname{pdet}\left(\mathcal{H}_{\mathrm{eff}}\right)$ is the pseudodeterminant of the Hamiltonian (the product of nonzero eigenvalues). The square root of the pseudodeterminant is in this case just the product of the positive eigenvalues (due to particle-hole symmetry). Since the system has only two non-negative single-particle energy levels $\left|E_{\uparrow}\right|=\left|B+E_{\varphi^{*}}\right|$ and $\left|E_{\downarrow}\right|=\mid B-E_{\varphi} *$, and one of these two energy levels vanishes at gapless points $\pm \varphi^{*}$ since in this case $|B|=\mid E_{\varphi} *$, the denominator of Equation 25 is equal to the nonzero positive energy level given by $|B|+\left|E_{\varphi^{*}}\right|=2|B|$, which yields $\sqrt{\left|\operatorname{pdet}\left(\mathcal{H}_{\text {eff }}\right)\right|}=2|B|$, which leads via Equation 25 to Equation 24.

Figure 4a shows the CPR of the SC-QD-SC junction for different choices of the Zeeman field $B$ at zero temperature, calculated directly from Equation 23. At low fields $|B|<B_{\min }$ (i.e., $\lambda>1)$ the system is topologically trivial $(P=1)$ and the CPR is smoothly oscillating without any discontinuity. At large fields $|B|>B_{\max }$ (i.e., $\lambda<-1$ ), the system is topologically nontrivial $(P=-1)$ and the Josephson current vanishes due to the opposite contribution of the two Andreev levels. At intermediate fields $B_{\min }<|B|<B_{\max }$ instead (i.e., $|\lambda|<1$ ), discontinuities appear at the transition points between the trivial and nontrivial topological states (gapless points $\pm \varphi^{*}$ ). The emergence of a discontinuous drop coincides with a change of the fermion parity and to the presence of zero-energy states closing the particle-hole gap. Since the energy levels of the system depends smoothly on the phase $\varphi$, gapless points are the only points where the CPR can be discontinuous. At finite temperatures, CPR discontinuities are smoothed out by the effect of thermal fluctuations. However, such discontinuities can be revealed, e.g., by the presence of spikes in the phase-derivative of the CPR at low temperatures [50].

Hence, if time-reversal symmetry is broken $(B \neq 0)$, current discontinuities correspond to the presence of zero-energy modes and to a change in the topological invariant. These signatures are topologically robust against small perturbations, such as disorder. This means that these discontinuities and the associated zero-energy modes cannot be removed by the presence of, e.g., disorder or interactions, if these perturbations are small compared to the effective local pairing $\Gamma$ and Zeeman energy $B$. The only effect of these small perturbations is in fact to produce a shift of the gapless point $\varphi^{*} \rightarrow \varphi^{*}+\delta \lambda / \sqrt{1-\lambda^{2}}$ where the topological transition and zero-energy modes occurs. Discontinuities in the Josephson CPR are still present in the interacting case [65] at zero temperature. As shown in [50], the correspondence between CPR discontinuities and fermion parity transitions relies only on the presence of a broken time-reversal symmetry that removes the spin degeneracy and on the fact that in this case the closing of the particle-hole gap correspond to a change of the topological invariant.

On the other hand, if time-reversal symmetry is unbroken, current discontinuities are still present if $B=\varepsilon=0$ (where $\lambda=1$ ). In this case, the CPR exhibits a single discontinuous drop $\Delta I=\Gamma / 2$ at the gapless point $\varphi^{*}=\pi$, according to Equation 24. This case reproduces the well-known current-phase discontinuity of a (a)

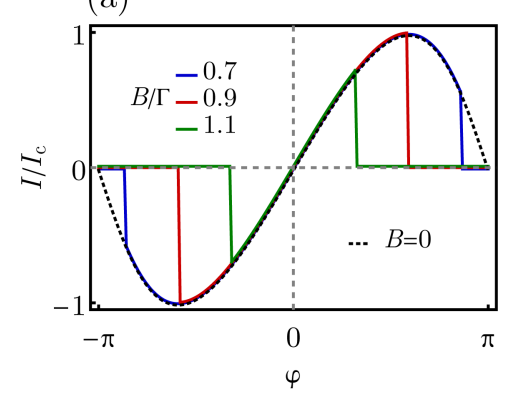

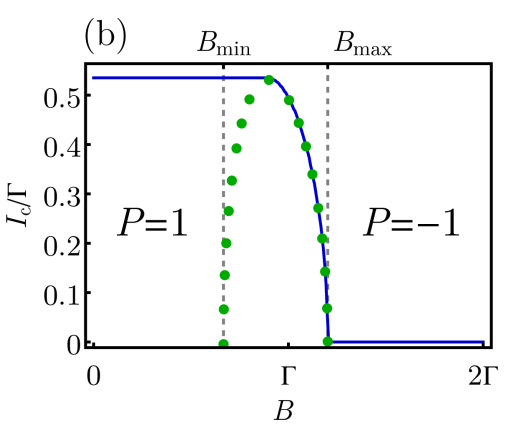

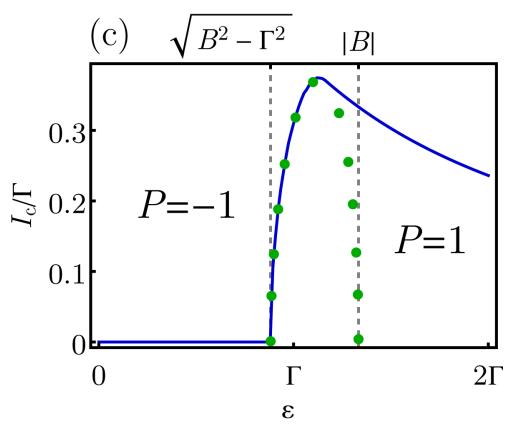

Figure 4: (a) Josephson CPR of the SC-QD-SC junction for different choices of the Zeeman field $B$ in the limit $T \rightarrow 0$ (Equation 23 ) in units of the critical current of the trivial branch. We take $\varepsilon=2 \Gamma / 3$. Depending on the Zeeman field, different regimes are realized: At small fields $|B|<B_{\text {min }}$ (i.e, $\lambda>1$, dotted line) the current is smoothly oscillating as a function of the phase $\varphi$ and the system is topologically trivial $(P=1)$. At large fields $|B|>B_{\text {max }}$ (i.e. $\lambda<-1$, not shown) the current vanishes and the system is topologically nontrivial $(P=-1)$. At intermediate fields $B_{\min }<|B|<B_{\text {max }}$ (i.e., $|\lambda|<1$, solid lines), discontinuous drops appear at the transition points between the trivial and nontrivial topological states. Current discontinuities correspond to the variations of the fermion parity and to the presence of zero energy modes. (b) Critical current of the SC-QD-SC junction as a function of the Zeeman field at zero temperature (solid line) with $\varepsilon=2 \Gamma / 3$. (c) Critical current of the SC-QD-SC junction as a function of the electric gate $\varepsilon$ at zero temperature (solid line) with $B=4 \Gamma / 3$. In both cases, the critical current drops from a finite value in the trivial state $(P=1$ and $\lambda>1)$ to zero in the nontrivial state $(P=-1$ and $\lambda<-1)$. In the transition regions $B_{\min }<B<B_{\max }$ (b) and $\sqrt{B^{2}-\Gamma^{2}}<|\varepsilon|<|B|$ (c), the trivial and nontrivial states alternate at different phases $\varphi$. As one can see, when the system approaches its nontrivial state $P=-1$, the critical current coincides with the magnitude of the discontinuous drop $\Delta /$ (green dots) given in Equation 24. 
quantum point contact [71]. However, in this case the discontinuity does not correspond to a topological transition.

The presence of a small Coulomb interaction does not affect the Josephson current at zero temperature in the trivial and nontrivial branches of the CPR, since the energy shift $U / 2$ of the Andreev levels do not depend on the phase $\varphi$.

\section{Critical current}

The topological transition can be probed also by a measure of the critical current of the junction. The critical current is defined as the maximum current of the junction up to the phase $I_{\mathrm{c}}=\max$ $I_{\varphi}$. In the trivial state at low fields $|B|<B_{\min }$ (i.e., $\lambda>1$ ) the critical current is finite. Since the CPR is continuous in this case, the maximum of the current coincides with the local maximum of the current where its phase-derivative vanishes $\partial_{\varphi} I_{\varphi}=0$. In the limits $\varepsilon \rightarrow 0$ and $\varepsilon \rightarrow \pm \Gamma$ for example, the current reaches its maximum at $\tilde{\varphi}=\pi$ or at $\tilde{\varphi}=\pi / 2$, which gives critical currents of $I_{\mathrm{c}}=\Gamma / 2$ and $I_{\mathrm{c}}=\Gamma^{2} /\left(4 \sqrt{\varepsilon^{2}+\Gamma^{2} / 2}\right)$, respectively. In the nontrivial state at large fields $|B|>B_{\max }$ instead $(\lambda<-1)$ the current vanishes and one has $I_{\mathrm{c}}=0$. However, at intermediate fields $B_{\min }<|B|<B_{\max }$ (i.e., $|\lambda|<1$ ) trivial and nontrivial states alternate in the interval $\varphi \in[-\pi, \pi]$, and the CPR has discontinuities. Because the CPR is not continuous, the maximum of the current may coincide either with the local maximum $I_{\tilde{\varphi}}$ of the current where $\partial_{\varphi} I_{\varphi}=0$, or with the current at the discontinuity $I_{\varphi^{*}}=\Delta I$. More precisely, the critical current coincides with the maximum between these two values $I_{\mathrm{c}}=\max \left(\left|I_{\tilde{\varphi}}\right|,|\Delta I|\right)$. The case $I_{\mathrm{c}}=|\Delta I|$ occurs, for instance, when the system approaches its nontrivial state at large fields $|B| \rightarrow B_{\max }$. Therefore for fields $|B| \leq B_{\max }$ the critical current coincides with the current discontinuity $I_{\mathrm{c}}=\Delta I$. This regime can be obtained either by a measure of the critical current by varying the magnetic field, or by varying, e.g., the energy level $\varepsilon$ in a constant field $B$.

Figure $4 \mathrm{~b}$ shows the critical current of the junction as a function of the Zeeman field. As one can see, the critical current is finite in the trivial $P=1$ state when $|B|<B_{\min }$ (i.e., $\lambda>1$ ), and drops to zero in the nontrivial $P=-1$ state when $|B|>B_{\max }$ (i.e., $\lambda<-1$ ) state. The drop of the critical current is smooth in the intermediate region where $B_{\min }<|B|<B_{\max }$ (i.e., $|\lambda|<1$ ). Analogously, Figure $4 \mathrm{c}$ shows the critical current of the junction as a function of the electric gate $\varepsilon$ at constant field $B$. The smooth transition is obtained for intermediate values $\sqrt{B^{2}-\Gamma^{2}}<\varepsilon<|B|$ the Zeeman field varies in the range $B_{\min }<|B|<B_{\max }$, where we remind that $B_{\min }=|\varepsilon|$ and $B_{\max }=\sqrt{\varepsilon^{2}+\Gamma^{2}}$. In the intermediate region, when the system approaches its nontrivial state, the critical current coincides with the magnitude of the discontinuous drop $I_{\mathrm{c}}=|\Delta I|$ (dots in the figures). Hence, a measure of the critical current at low temper- ature can be used to indirectly probe the magnitude of the discontinuous drop and the existence of topological phase transitions and zero-energy modes even when a direct measure of the CPR is not accessible [72]. It is reasonable to speculate that the current discontinuities may indicate a topological transition also in the interacting case.

\section{Conclusion}

We have shown that a quantum dot coupled with two superconducting leads can realize a $0 \mathrm{D}$ topological superconductor with broken time-reversal symmetry. In this system, topological phase transitions between trivial and nontrivial states correspond to discontinuities in the Josephson CPR at low temperatures and to the presence of zero-energy modes. This simple model, which can be treated analytically, fully confirms the results obtained in a more general model in [50].

The topological phase transitions and the ensuing current discontinuities are robust, in the sense that cannot be removed by small perturbations. A direct measure of the CPR [71,73-75] or of the Josephson radiation $[38,76,77]$ at low temperatures can reveal the presence of such discontinuities. Moreover, the presence of the topological transition can be probed indirectly by a measure of the critical current of the junction as a function of the Zeeman field or gate voltage.

\section{Acknowledgements}

P. M. acknowledges financial support from JSPS Kakenhi Grant No. 16H02204. A. B. acknowledges the Italian MIUR-FIRB 2012 via the HybridNanoDev project under Grant no. RBFR1236VV, the European Research Council under the European Union's Seventh Framework Program (FP7/ 2007-2013) ERC Grant No. 615187-COMANCHE for partial financial support, the CNR-CONICET cooperation programme "Energy conversion in quantum nanoscale hybrid devices", and the Royal Society though the International Exchanges between the UK and Italy (grant IES R3 170054).

\section{ORCID ${ }^{\circledR}$ iDs}

Pasquale Marra - https://orcid.org/0000-0002-9545-3314

Alessandro Braggio - https://orcid.org/0000-0003-2119-1160

Roberta Citro - https://orcid.org/0000-0002-3896-4759

\section{References}

1. v. Klitzing, K.; Dorda, G.; Pepper, M. Phys. Rev. Lett. 1980, 45, 494-497. doi:10.1103/PhysRevLett.45.494

2. Thouless, D. J.; Kohmoto, M.; Nightingale, M. P.; den Nijs, M. Phys. Rev. Lett. 1982, 49, 405-408. doi:10.1103/PhysRevLett.49.405

3. Kitaev, A. Yu. Phys.-Usp. 2001, 44, 131. doi:10.1070/1063-7869/44/10S/S29

4. Hasan, M. Z.; Kane, C. L. Rev. Mod. Phys. 2010, 82, 3045-3067. doi:10.1103/RevModPhys.82.3045 
5. Qi, X.-L.; Zhang, S.-C. Rev. Mod. Phys. 2011, 83, 1057-1110. doi:10.1103/RevModPhys.83.1057

6. Wehling, T. O.; Black-Schaffer, A. M.; Balatsky, A. V. Adv. Phys. 2014, 63, 1-76. doi:10.1080/00018732.2014.927109

7. Chiu, C.-K.; Teo, J. C. Y.; Schnyder, A. P.; Ryu, S. Rev. Mod. Phys. 2016, 88, 035005. doi:10.1103/RevModPhys.88.035005

8. Altland, A.; Zirnbauer, M. R. Phys. Rev. B 1997, 55, 1142-1161. doi:10.1103/PhysRevB.55.1142

9. Schnyder, A. P.; Ryu, S.; Furusaki, A.; Ludwig, A. W. W. AIP Conf. Proc. 2009, 1134, 10-21. doi:10.1063/1.3149481

10. Kitaev, A. AIP Conf. Proc. 2009, 1134, 22-30. doi:10.1063/1.3149495

11. Mourik, V.; Zuo, K.; Frolov, S. M.; Plissard, S. R.; Bakkers, E. P. A. M.; Kouwenhoven, L. P. Science 2012, 336, 1003-1007. doi:10.1126/science.1222360

12. Nadj-Perge, S.; Drozdov, I. K.; Li, J.; Chen, H.; Jeon, S.; Seo, J.; MacDonald, A. H.; Bernevig, B. A.; Yazdani, A. Science 2014, 346, 602-607. doi:10.1126/science.1259327

13. Pawlak, R.; Kisiel, M.; Klinovaja, J.; Meier, T.; Kawai, S.; Glatzel, T.; Loss, D.; Meyer, E. npj Quantum Inf. 2016, 2, 16035. doi:10.1038/npjqi.2016.35

14. Alicea, J. Rep. Prog. Phys. 2012, 75, 076501. doi:10.1088/0034-4885/75/7/076501

15. Aguado, R. Riv. Nuovo Cimento Soc. Ital. Fis. 2017, 40, 523. doi:10.1393/ncr/i2017-10141-9

16. Lutchyn, R. M.; Bakkers, E. P. A. M.; Kouwenhoven, L. P.; Krogstrup, P.; Marcus, C. M.; Oreg, Y. Nat. Rev. Mater. 2018, 3, 52-68. doi:10.1038/s41578-018-0003-1

17. Sato, M.; Ando, Y. Rep. Prog. Phys. 2017, 80, 076501. doi:10.1088/1361-6633/aa6ac7

18. Leijnse, M.; Flensberg, K. Semicond. Sci. Technol. 2012, 27, 124003. doi:10.1088/0268-1242/27/12/124003

19. Elliott, S. R.; Franz, M. Rev. Mod. Phys. 2015, 87, 137-163. doi:10.1103/RevModPhys.87.137

20. Beenakker, C. W. J. Annu. Rev. Condens. Matter Phys. 2013, 4, 113-136. doi:10.1146/annurev-conmatphys-030212-184337

21. Lutchyn, R. M.; Sau, J. D.; Das Sarma, S. Phys. Rev. Lett. 2010, 105, 077001. doi:10.1103/PhysRevLett.105.077001

22. Oreg, Y.; Refael, G.; von Oppen, F. Phys. Rev. Lett. 2010, 105, 177002. doi:10.1103/PhysRevLett.105.177002

23. Gangadharaiah, S.; Braunecker, B.; Simon, P.; Loss, D. Phys. Rev. Lett. 2011, 107, 036801. doi:10.1103/PhysRevLett.107.036801

24. Klinovaja, J.; Stano, P.; Loss, D. Phys. Rev. Lett. 2012, 109, 236801. doi:10.1103/PhysRevLett.109.236801

25. Klinovaja, J.; Stano, P.; Yazdani, A.; Loss, D. Phys. Rev. Lett. 2013, 111, 186805. doi:10.1103/PhysRevLett.111.186805

26. Jiang, L.; Pekker, D.; Alicea, J.; Refael, G.; Oreg, Y.; von Oppen, F. Phys. Rev. Lett. 2011, 107, 236401. doi:10.1103/PhysRevLett.107.236401

27. San-Jose, P.; Prada, E.; Aguado, R. Phys. Rev. Lett. 2012, 108, 257001. doi:10.1103/PhysRevLett.108.257001

28. Rokhinson, L. P.; Liu, X.; Furdyna, J. K. Nat. Phys. 2012, 8, 795-799. doi:10.1038/nphys2429

29. Brunetti, A.; Zazunov, A.; Kundu, A.; Egger, R. Phys. Rev. B 2013, 88, 144515. doi:10.1103/PhysRevB.88.144515

30. Chang, W.; Manucharyan, V. E.; Jespersen, T. S.; Nygård, J.; Marcus, C. M. Phys. Rev. Lett. 2013, 110, 217005. doi:10.1103/PhysRevLett.110.217005

31. Dolcini, F.; Houzet, M.; Meyer, J. S. Phys. Rev. B 2015, 92, 035428. doi:10.1103/PhysRevB.92.035428
32. Khanna, U.; Mukherjee, D. K.; Kundu, A.; Rao, S. Phys. Rev. B 2016, 93, 121409. doi:10.1103/PhysRevB.93.121409

33. Peng, Y.; Pientka, F.; Berg, E.; Oreg, Y.; von Oppen, F. Phys. Rev. B 2016, 94, 085409. doi:10.1103/PhysRevB.94.085409

34. Hussein, R.; Jaurigue, L.; Governale, M.; Braggio, A. Phys. Rev. B 2016, 94, 235134. doi:10.1103/PhysRevB.94.235134

35. Mellars, E.; Béri, B. Phys. Rev. B 2016, 94, 174508. doi:10.1103/PhysRevB.94.174508

36. Wiedenmann, J.; Bocquillon, E.; Deacon, R. S.; Hartinger, S.; Herrmann, O.; Klapwijk, T. M.; Maier, L.; Ames, C.; Brüne, C.; Gould, C.; Oiwa, A.; Ishibashi, K.; Tarucha, S.; Buhmann, H.; Molenkamp, L. W. Nat. Commun. 2016, 7, 10303. doi:10.1038/ncomms10303

37. Jellinggaard, A.; Grove-Rasmussen, K.; Madsen, M. H.; Nygård, J. Phys. Rev. B 2016, 94, 064520. doi:10.1103/PhysRevB.94.064520

38. Deacon, R. S.; Wiedenmann, J.; Bocquillon, E.; Domínguez, F.; Klapwijk, T. M.; Leubner, P.; Brüne, C.; Hankiewicz, E. M.; Tarucha, S.; Ishibashi, K.; Buhmann, H.; Molenkamp, L. W. Phys. Rev. X 2017, 7, 021011. doi:10.1103/PhysRevX.7.021011

39. Virtanen, P.; Bergeret, F. S.; Strambini, E.; Giazotto, F.; Braggio, A. arXiv 2017, No. 1712.01684.

40. Mukherjee, D. K.; Rao, S.; Kundu, A. Phys. Rev. B 2017, 96, 161408. doi:10.1103/PhysRevB.96.161408

41. Khanna, U.; Rao, S.; Kundu, A. Phys. Rev. B 2017, 95, 201115. doi:10.1103/PhysRevB.95.201115

42. Pientka, F.; Keselman, A.; Berg, E.; Yacoby, A.; Stern, A.; Halperin, B. I. Phys. Rev. X 2017, 7, 021032. doi:10.1103/PhysRevX.7.021032

43. Huang, H.; Liang, Q.-F.; Yao, D.-X.; Wang, Z. Phys. C (Amsterdam, Neth.) 2017, 543, 22-26. doi:10.1016/j.physc.2017.10.005

44. Lee, E. J. H.; Jiang, X.; Žitko, R.; Aguado, R.; Lieber, C. M.; De Franceschi, S. Phys. Rev. B 2017, 95, 180502. doi:10.1103/PhysRevB.95.180502

45. Cayao, J.; San-Jose, P.; Black-Schaffer, A. M.; Aguado, R.; Prada, E. Phys. Rev. B 2017, 96, 205425. doi:10.1103/PhysRevB.96.205425

46. San-Jose, P.; Cayao, J.; Prada, E.; Aguado, R. New J. Phys. 2013, 15 , 075019. doi:10.1088/1367-2630/15/7/075019

47. Cayao, J.; Black-Schaffer, A. M.; Prada, E.; Aguado, R. Beilstein J. Nanotechnol. 2018, 9, 1339-1357. doi:10.3762/bjnano.9.127

48. Domínguez, F.; Kashuba, O.; Bocquillon, E.; Wiedenmann, J.; Deacon, R. S.; Klapwijk, T. M.; Platero, G.; Molenkamp, L. W.; Trauzettel, B.; Hankiewicz, E. M. Phys. Rev. B 2017, 95, 195430. doi:10.1103/PhysRevB.95.195430

49. Zyuzin, A.; Alidoust, M.; Loss, D. Phys. Rev. B 2016, 93, 214502. doi:10.1103/PhysRevB.93.214502

50. Marra, P.; Citro, R.; Braggio, A. Phys. Rev. B 2016, 93, 220507. doi:10.1103/PhysRevB.93.220507

51. Loss, D.; DiVincenzo, D. P. Phys. Rev. A 1998, 57, 120-126. doi:10.1103/PhysRevA.57.120

52. Choi, M.-S.; Bruder, C.; Loss, D. Phys. Rev. B 2000, 62, 13569-13572. doi:10.1103/PhysRevB.62.13569

53. Kouwenhoven, L. P.; Austing, D. G.; Tarucha, S. Rep. Prog. Phys. 2001, 64, 701. doi:10.1088/0034-4885/64/6/201

54. van der Wiel, W. G.; Fujisawa, T.; Tarucha, S.; Kouwenhoven, L. P. Jpn. J. Appl. Phys. 2001, 40, 2100. doi:10.1143/JJAP.40.2100

55. Tarasinski, B.; Chevallier, D.; Hutasoit, J. A.; Baxevanis, B.; Beenakker, C. W. J. Phys. Rev. B 2015, 92, 144306. doi:10.1103/PhysRevB.92.144306 
56. Beenakker, C. W. J.; Edge, J. M.; Dahlhaus, J. P.; Pikulin, D. I.; Mi, S.; Wimmer, M. Phys. Rev. Lett. 2013, 111, 037001.

doi:10.1103/PhysRevLett.111.037001

57. Stanescu, T. D.; Lutchyn, R. M.; Das Sarma, S. Phys. Rev. B 2013, 87, 094518. doi:10.1103/PhysRevB.87.094518

58. Lee, E. J. H.; Jiang, X.; Houzet, M.; Aguado, R.; Lieber, C. M.; De Franceschi, S. Nat. Nanotechnol. 2013, 9, 79-84. doi:10.1038/nnano.2013.267

59. Yokoyama, T.; Eto, M.; Nazarov, Y. V. J. Phys. Soc. Jpn. 2013, 82, 054703. doi:10.7566/JPSJ.82.054703

60. Yokoyama, T.; Eto, M.; Nazarov, Y. V. Phys. Rev. B 2014, 89, 195407. doi:10.1103/PhysRevB.89.195407

61. Klees, R. L.; Rastelli, G.; Belzig, W. Phys. Rev. B 2017, 96, 144510. doi:10.1103/PhysRevB.96.144510

62. Li, Y.; Kundu, A.; Zhong, F.; Seradjeh, B. Phys. Rev. B 2014, 90 , 121401. doi:10.1103/PhysRevB.90.121401

63. Benito, M.; Platero, G. Phys. E (Amsterdam, Neth.) 2015, 74, 608-613. doi:10.1016/j.physe.2015.08.030

64. Meng, T.; Florens, S.; Simon, P. Phys. Rev. B 2009, 79, 224521. doi:10.1103/PhysRevB.79.224521

65. Wentzell, N.; Florens, S.; Meng, T.; Meden, V.; Andergassen, S. Phys. Rev. B 2016, 94, 085151. doi:10.1103/PhysRevB.94.085151

66. Braggio, A.; Governale, M.; Pala, M. G.; König, J. Solid State Commun. 2011, 151, 155-158. doi:10.1016/j.ssc.2010.10.043

67. Droste, S.; Andergassen, S.; Splettstoesser, J. J. Phys.: Condens. Matter 2012, 24, 415301. doi:10.1088/0953-8984/24/41/415301

68. Hussein, R.; Braggio, A.; Governale, M. Phys. Status Solidi B 2017, 254, 1600603. doi:10.1002/pssb.201600603

69. Nazarov, Y. V.; Blanter, Y. M. Quantum Transport: Introduction to Nanoscience; Cambridge University Press, 2009; p 106.

70. Loring, T. A. Ann. Phys. 2015, 356, 383-416. doi:10.1016/j.aop.2015.02.031

71. Golubov, A. A.; Kupriyanov, M. Y.; Il'ichev, E. Rev. Mod. Phys. 2004, 76, 411-469. doi:10.1103/RevModPhys.76.411

72. Tiira, J.; Strambini, E.; Amado, M.; Roddaro, S.; San-Jose, P.; Aguado, R.; Bergeret, F. S.; Ercolani, D.; Sorba, L.; Giazotto, F. Nat. Commun. 2017, 8, 14984. doi:10.1038/ncomms14984

73. Frolov, S. M.; Van Harlingen, D. J.; Oboznov, V. A.; Bolginov, V. V.; Ryazanov, V. V. Phys. Rev. B 2004, 70, 144505. doi:10.1103/PhysRevB.70.144505

74. Sochnikov, I.; Bestwick, A. J.; Williams, J. R.; Lippman, T. M.; Fisher, I. R.; Goldhaber-Gordon, D.; Kirtley, J. R.; Moler, K. A. Nano Lett. 2013, 13, 3086-3092. doi:10.1021/nl400997k

75. Szombati, D. B.; Nadj-Perge, S.; Car, D.; Plissard, S. R.; Bakkers, E. P. A. M.; Kouwenhoven, L. P. Nat. Phys. 2016, 12, 568-572. doi:10.1038/nphys3742

76. Ozyuzer, L.; Koshelev, A. E.; Kurter, C.; Gopalsami, N.; Li, Q.; Tachiki, M.; Kadowaki, K.; Yamamoto, T.; Minami, H.; Yamaguchi, H.; Tachiki, T.; Gray, K. E.; Kwok, W.-K.; Welp, U. Science 2007, 318, 1291-1293. doi:10.1126/science.1149802

77. Solinas, P.; Gasparinetti, S.; Golubev, D.; Giazotto, F. Sci. Rep. 2015, 5, 12260. doi:10.1038/srep12260

\section{License and Terms}

This is an Open Access article under the terms of the Creative Commons Attribution License

(http://creativecommons.org/licenses/by/4.0), which permits unrestricted use, distribution, and reproduction in any medium, provided the original work is properly cited.

The license is subject to the Beilstein Journal of Nanotechnology terms and conditions:

(https://www.beilstein-journals.org/bjnano)

The definitive version of this article is the electronic one which can be found at:

doi:10.3762/bjnano.9.162 